\title{
Gravitational lensing and frame-dragging of light in the Kerr-Newman and the Kerr-Newman-(anti) de Sitter black hole spacetimes
}

\author{
G. V. Kraniotis*i \\ University of Ioannina, Physics Department, Section of Theoretical Physics \\ Ioannina, Greece GR 45110 \\ E-mail: gkraniotecc.uoi.gr
}

\begin{abstract}
The null geodesics that describe photon orbits in the curved spacetime of a rotating electrically charged black hole (Kerr-Newman) are solved exactly including the contribution from the cosmological constant. Thus the theory produced in this work is a complete theory for the propagation of light signals in the field of rotating charged black holes: all of its fundamental parameters enter the analytic solutions on an equal footing. We first solve exactly null geodesics on the sphere and produce closed form solutions for the frame dragging of light for spherical polar and non-polar light orbits in the Kerr-Newman-(anti)de Sitter (KN(a)dS) black hole spacetime. Our solutions are expressed elegantly in terms of multivariable hypergeometric functions of Appell-Lauricella and Weierstraßelliptic function. We then derive the closed form analytic solution for the deflection angle that an equatorial unbound light ray undergoes by a Kerr-Newman (KN) black hole. Various limiting cases are studied. In particular the deflection angle for a non-spinning charged ReisserNordström black hole is computed analytically in terms of Appell's hypergeometric function $F_{1}$ or in terms of inverse Jacobian functions. The more involved problem of treating a KN black hole as a gravitational lens, i.e. a KN black hole along with a static source of light and a static observer both located far away but otherwise at arbitrary positions in space is solved analytically. For this model, we derive the analytic solutions of the lens equations in terms of Appell and Lauricella hypergeometric functions and the Weierstraß modular form.
\end{abstract}

18th International Conference From the Planck Scale to the Electroweak Scale 25-29 May 2015

Ioannina, Greece

\footnotetext{
*Speaker.

${ }^{\dagger}$ Contributed talk, the author thanks the organisers for their kind invitation and for organising such an exciting event. Work partially supported by research grant:THALIS "Beyond the Standard Model:Theoretical Physics of Elementary Particles and Cosmology under the light of LHC”, MIS 375734, code E AKE 80803.
} 


\section{Motivation}

In this talk I am going to review recent results of mine, which are published in [2], in which I have obtained closed form analytic solutions of the null geodesics in the spacetime surrounding an electrically charged, rotating cosmological black hole, namely the Kerr-Newman-(anti) de Sitter black hole. The KN(a)dS)metric is the most general exact stationary black hole solution of the Einstein-Maxwell system of differential equations. The system of geodesics in the KN(a)dS black hole $(\mathrm{BH})$ solution is a completely integrable dynamical system.

The metric in Boyer-Lindquist coordinates is [1]:

$$
\begin{gathered}
\mathrm{d} s^{2}=\frac{\Delta_{r}^{K N}}{\Xi^{2} \rho^{2}}\left(c \mathrm{~d} t-a \sin ^{2} \theta \mathrm{d} \phi\right)^{2}-\frac{\rho^{2}}{\Delta_{r}^{K N}} \mathrm{~d} r^{2}-\frac{\rho^{2}}{\Delta_{\theta}} \mathrm{d} \theta^{2}-\frac{\Delta_{\theta} \sin ^{2} \theta}{\Xi^{2} \rho^{2}}\left(a c \mathrm{~d} t-\left(r^{2}+a^{2}\right) \mathrm{d} \phi\right)^{2} \\
\Delta_{\theta}:=1+\frac{a^{2} \Lambda}{3} \cos ^{2} \theta, \Xi:=1+\frac{a^{2} \Lambda}{3}, \rho^{2}=r^{2}+a^{2} \cos ^{2} \theta \\
\Delta_{r}^{K N}:=\left(1-\frac{\Lambda}{3} r^{2}\right)\left(r^{2}+a^{2}\right)-2 \frac{G M}{c^{2}} r+\frac{G e^{2}}{c^{4}},
\end{gathered}
$$

where $a, M$, $e$ denote the Kerr parameter (spin), mass and electric charge of the black hole respectively, while $\Lambda$ is the cosmological constant. Also, $G, c$ denote Newton's gravitational constant and speed of light respectively.

This is accompanied by a non-zero electromagnetic field $F=\mathrm{d} A$ with vector potential $(G=$ $c=1)$ :

$$
A=-\frac{e r}{\Xi\left(r^{2}+a^{2} \cos ^{2} \theta\right)}\left(\mathrm{d} t-a \sin ^{2} \theta \mathrm{d} \phi\right) .
$$

For the surrounding spacetime to represent a black hole, i.e. the singularity surrounded by the horizon, the electric charge and angular momentum $J$ must be restricted by the relation:

$$
\begin{gathered}
\frac{G M}{c^{2}} \geq\left[\left(\frac{J}{M c}\right)^{2}+\frac{G e^{2}}{c^{4}}\right]^{1 / 2} \Leftrightarrow \\
\frac{G M}{c^{2}} \geq\left[a^{2}+\frac{G e^{2}}{c^{4}}\right]^{1 / 2} \Rightarrow e^{2} \leq G M^{2}\left(1-a^{\prime 2}\right)
\end{gathered}
$$

The dynamical system of geodesic equations in $\mathrm{KN}(\mathrm{a}) \mathrm{dS}$ spacetime is completely integrable and the geodesic equations can be derived by solving the relativistic Hamilton-Jacobi equation by separation of variables [2]:

$$
\begin{aligned}
\int \frac{\mathrm{d} r}{\sqrt{R^{\prime}}} & =\int \frac{\mathrm{d} \theta}{\sqrt{\Theta^{\prime}}}, \rho^{2} \frac{\mathrm{d} r}{\mathrm{~d} \lambda}= \pm \sqrt{R^{\prime}}, \rho^{2} \frac{\mathrm{d} \theta}{\mathrm{d} \lambda}= \pm \sqrt{\Theta^{\prime}}, \\
\rho^{2} \frac{\mathrm{d} \phi}{\mathrm{d} \lambda} & =-\frac{\Xi^{2}}{\Delta_{\theta} \sin ^{2} \theta}\left(a E \sin ^{2} \theta-L\right)+\frac{a \Xi^{2}}{\Delta_{r}^{K N}}\left[\left(r^{2}+a^{2}\right) E-a L\right], \\
c \rho^{2} \frac{\mathrm{d} t}{\mathrm{~d} \lambda} & =\frac{\Xi^{2}\left(r^{2}+a^{2}\right)\left[\left(r^{2}+a^{2}\right) E-a L\right]}{\Delta_{r}^{K N}}-\frac{a \Xi^{2}\left(a E \sin ^{2} \theta-L\right)}{\Delta_{\theta}},
\end{aligned}
$$


where

$$
\begin{aligned}
& R^{\prime}:=\Xi^{2}\left[\left(r^{2}+a^{2}\right) E-a L\right]^{2}-\Delta_{r}^{K N}\left(\mu^{2} r^{2}+Q+\Xi^{2}(L-a E)^{2}\right), \\
& \Theta^{\prime}:=\left[Q+(L-a E)^{2} \Xi^{2}-\mu^{2} a^{2} \cos ^{2} \theta\right] \Delta_{\theta}-\Xi^{2} \frac{\left(a E \sin ^{2} \theta-L\right)^{2}}{\sin ^{2} \theta} .
\end{aligned}
$$

Null geodesics are derived by setting $\mu=0$. The quantities $E, Q, L$ are first integrals of motion. We use geometrized units, $G=c=1$, unless it is stipulated otherwise.

\section{Null geodesics on the sphere and frame dragging of light}

The relevant differential equation is:

$$
\frac{\mathrm{d} \phi}{\mathrm{d} \theta}=\frac{\frac{a P}{\Delta^{K N}}-a E+\frac{L}{\sin ^{2} \theta}}{\sqrt{Q-\cos ^{2} \theta\left[-a^{2} E^{2}+\frac{L^{2}}{\sin ^{2} \theta}\right]}}
$$

where $\Delta^{K N}:=r^{2}+a^{2}+e^{2}-2 M r$, assuming $\Lambda=0$ and a constant radius. It is convenient to introduce the parameters:

$$
\Phi:=L / E, \mathscr{Q}:=Q / E^{2}
$$

Using the variable: $z:=\cos ^{2} \theta$

$$
\mathrm{d} \phi=-\frac{1}{2} \frac{\mathrm{d} z}{\sqrt{\alpha z^{3}-z^{2}(\alpha+\beta)+\mathscr{Q} z}} \times\left\{\frac{a P}{\Delta^{K N}}-a+\frac{\Phi}{1-z}\right\}
$$

where

$$
\alpha:=-a^{2}, \quad \beta:=\mathscr{Q}+\Phi^{2} .
$$

Assuming $\Phi=0$ and using

$$
z:=-\frac{\xi+\frac{\alpha+\beta}{12}}{-\alpha / 4}
$$

we obtain the integral equation:

$$
\int \mathrm{d} \phi=\int \frac{1}{2} \frac{\mathrm{d} \xi}{\sqrt{4 \xi^{3}-g_{2} \xi-g_{3}}} \times\left\{\frac{a P^{\prime}}{\Delta^{K N}}-a\right\}
$$

and this orbit integral can be inverted by the Weierstraß modular Jacobi form

$$
\xi=\wp\left(\frac{-\phi+\varepsilon}{A}\right)
$$

where $A:=-\frac{1}{2}\left(\frac{a P^{\prime}}{\Delta^{K N}}-a\right), P^{\prime}=\left(r^{2}+a^{2}\right)$ and the Weierstraß invariants take the form

$$
g_{2}=\frac{1}{12}(\alpha+\beta)^{2}-\frac{\mathscr{Q} \alpha}{4}, g_{3}=\frac{1}{216}(\alpha+\beta)^{3}-\frac{\mathscr{Q} \alpha^{2}}{48}-\frac{\mathscr{Q} \alpha \beta}{48} .
$$

Now the closed form solution for the spherical polar orbit $(\Phi=0)$ is: [2] 
Theorem 1.

$$
\wp(-\phi+\varepsilon)=\frac{\alpha^{\prime \prime}}{4} \cos ^{2} \theta-\frac{1}{12}\left(\alpha^{\prime \prime}+\beta^{\prime \prime}\right)=\tilde{\xi}:=\frac{\xi}{A^{2}},
$$

where $\alpha^{\prime \prime}:=\alpha^{\prime} / A^{\prime 2}=\frac{\alpha}{a^{2} A^{\prime 2}}=-\frac{1}{A^{\prime 2}}, \beta^{\prime \prime}:=\beta^{\prime} / A^{\prime 2}=\frac{\mathscr{Q}}{a^{2} A^{\prime 2}}, \mathscr{Q}^{\prime \prime}=\mathscr{Q}^{\prime} / A^{\prime 2}=\frac{\mathscr{Q}}{a^{2} A^{\prime 2}}$ and $\varepsilon$ denotes a constant of integration. Also $A^{\prime}$ is given by the expression:

$$
A^{\prime}:=\frac{\frac{e^{2}}{2 a^{2}}-\frac{M r}{a^{2}}}{\frac{r^{2}}{a^{2}}+\frac{e^{2}}{a^{2}}+1-\frac{2 M r}{a^{2}}} .
$$

Explicitely eqn (2.9) reads: $\tilde{\xi}=\wp\left(-\phi+\wp^{-1}\left(\tilde{\xi}_{0}\right)\right)$, where $\tilde{\xi}_{0}$ is the initial value of $\tilde{\xi}$. The Weierstraßinvariants are:

$$
\begin{aligned}
& g_{2}^{\prime \prime}=\frac{1}{12}\left(\alpha^{\prime \prime}+\beta^{\prime \prime}\right)^{2}-\frac{\mathscr{Q}^{\prime \prime} \alpha^{\prime \prime}}{4}=\frac{1}{12} \frac{\left(-a^{2}+\mathscr{Q}\right)^{2}}{a^{4} A^{\prime 4}}+\frac{\mathscr{Q}}{4 a^{2} A^{14}}, \\
& g_{3}^{\prime \prime}=\frac{1}{432 a^{6} A^{\prime 6}}\left[-2 a^{6}-3 a^{4} \mathscr{Q}+3 a^{2} \mathscr{Q}^{2}+2 \mathscr{Q}^{3}\right] .
\end{aligned}
$$

The assumption of spherical orbits, results in two conditions from the vanishing of the polynomial $R(r)$ and its first derivative:

$$
\Phi=\frac{a^{2} M+a^{2} r+2 e^{2} r-3 M r^{2}+r^{3}}{a(M-r)}, \mathscr{Q}=\frac{-r^{2}\left[4 a^{2}\left(e^{2}-M r\right)+\left(2 e^{2}+r(-3 M+r)\right)^{2}\right]}{a^{2}(M-r)^{2}}
$$

which are also the conditions for the photon to escape to infinity. After a complete oscillation in latitude, the angle of longitude, which determines the amount of dragging for the spherical photon polar orbit in the general theory of relativity (GTR) for the KN black hole, increases by:

\section{Theorem 2.}

$$
\begin{gathered}
\Delta \phi_{p K N}^{G T R}=4 \omega, \\
\omega=\frac{1}{\sqrt{\frac{\left(a^{2}+\mathscr{Q}\right)\left(a^{2}+e^{2}+(-2+r) r\right)^{2}}{a^{2}\left(e^{2}-2 r\right)^{2}}}} \frac{\pi}{2} F\left(\frac{1}{2}, \frac{1}{2}, 1, \frac{a^{2}}{a^{2}+\mathscr{Q}}\right),
\end{gathered}
$$

where $\omega$ is the real half-period of the Weierstraß elliptic function $\wp$, and $F(\alpha, \beta, \gamma, z)$ denotes the Gauß hypergeometric function. The generalisation of Theorem 2 in the presense of $\Lambda$ is [2]:

Theorem 3. The closed form solution for the frame dragging (Lense-Thirring effect) of light that a null spherical polar undergoes in the spacetime of a Kerr-Newman-(anti)de Sitter black hole is [2]:

$$
\begin{gathered}
\Delta \phi_{p K N \Lambda}^{G T R}=-\frac{\Xi^{2} a}{2} \frac{1}{\sqrt{\mathscr{Q}}} F_{1}\left(\frac{1}{2}, 1, \frac{1}{2}, 1,-\frac{a^{2} \Lambda}{3},-\frac{\mathscr{Q} a^{2} \frac{\Lambda}{3}+a^{2} \Xi^{3}}{\mathscr{Q}}\right) \frac{\Gamma^{2}(1 / 2)}{\Gamma(1)}+ \\
\frac{a \Xi^{2}\left(r^{2}+a^{2}\right)}{\Delta_{r}^{K N} \sqrt{\mathscr{Q}}} \frac{1}{2} F\left(\frac{1}{2}, \frac{1}{2}, 1,-\frac{\mathscr{Q} a^{2} \frac{\Lambda}{3}+a^{2} \Xi^{3}}{\mathscr{Q}}\right) \frac{\Gamma^{2}(1 / 2)}{\Gamma(1)} .
\end{gathered}
$$

where $F_{1}\left(\alpha, \beta, \beta^{\prime}, \gamma, x, y\right)$ is Appell's first hypergeometric function of two variables $x, y$ and parameters $\alpha, \beta, \beta^{\prime}, \gamma$ [4]. The generalisation of Theorem 3 to the case of spherical non-polar $(\Phi \neq 0)$ orbits in $\mathrm{KN}(\mathrm{a}) \mathrm{dS}$ spacetime is [2]: 
Theorem 4. The exact amount of frame dragging for a spherical non polar light orbit in the $K N($ a)dS spacetime is:

$$
\begin{aligned}
\Delta \phi_{n P K N \Lambda}^{G T R} & =4\left[\frac{a \Xi^{2}}{\Delta_{r}^{K N}} \frac{\left[\left(r^{2}+a^{2}\right)-a \Phi\right]}{|H|} \sqrt{\frac{1}{z_{m}-z_{3}}} \frac{\pi}{2} F\left(\frac{1}{2}, \frac{1}{2}, 1, \frac{z_{m}}{z_{m}-z_{3}}\right)\right. \\
& +\frac{\Xi^{2} \Phi}{2|H|} \frac{z_{m}}{\left(1-\eta z_{m}\right)\left(1-z_{m}\right)} \frac{1}{\sqrt{z_{m}^{2}\left(z_{m}-z_{3}\right)}} F_{D}\left(\frac{1}{2}, \beta_{4}^{\Lambda 1}, \frac{3}{2}, z_{\Lambda 0}^{\alpha_{1}}\right) 2 \\
& \left.-\frac{\Xi^{2} a}{2|H|} \frac{z_{m}}{\sqrt{z_{m}^{2}\left(z_{m}-z_{3}\right)}} \frac{1}{1-\eta z_{m}} F_{D}\left(\frac{1}{2}, \beta_{3}^{4}, \frac{3}{2}, z_{\Lambda 0}^{\alpha_{2}}\right) 2\right] .
\end{aligned}
$$

The parameters and variables of Lauricella's function $F_{D}$ which appears in the analytic solution of Theorem 4 are defined in Eqs.(52)-(54) in [2]. Also $H^{2}:=\frac{a^{2} \Lambda}{3}\left[\mathscr{Q}+(\Phi-a)^{2} \Xi^{2}\right]+a^{2} \Xi^{2}$.

\section{Closed form analytic computation for the deflection angle of an equatorial unbound light ray in the Kerr-Newman spacetime}

For equatorial geodesics the parameter $\mathscr{Q}=0$ and the relevant differential equation for the exact computation of the deflection angle is:

$$
\frac{\mathrm{d} \phi}{\mathrm{d} r}=\frac{e^{2}(\Phi-a)+\Phi r^{2}-2 M r(\Phi-a)}{(\underbrace{r^{2}+a^{2}+e^{2}-2 M r}_{=: \Delta^{K N}}) \sqrt{R}},
$$

where the quartic radial polynomial has the form:

$$
R=r^{4}+r^{2}\left(a^{2}-\Phi^{2}\right)+2 M r\left(a^{2}+\Phi^{2}-2 \Phi a\right)-e^{2}(\Phi-a)^{2} .
$$

We compute the following integral applying the partial fractions technique:

$$
\begin{aligned}
\int \mathrm{d} \phi & =\int \frac{e^{2}(\Phi-a)+\Phi r^{2}-2 M r(\Phi-a)}{\Delta^{K N} \sqrt{R}} \mathrm{~d} r \\
& =\int \frac{\Phi}{\sqrt{R}} \mathrm{~d} r+\int \frac{A_{+}^{e q K N}}{\left(r-r_{+}\right) \sqrt{R}} \mathrm{~d} r+\int \frac{A_{-}^{e q K N}}{\left(r-r_{-}\right) \sqrt{R}} \mathrm{~d} r
\end{aligned}
$$

Thus $\Delta \phi_{e K N}^{G T R}=2 \int_{\alpha}^{\infty}$. We organize all roots in ascending order of magnitude as follows,

$$
\alpha_{\mu}>\alpha_{v}>\alpha_{i}>\alpha_{\rho}
$$

where $\alpha_{\mu}=\alpha_{\mu+1}, \alpha_{v}=\alpha_{\mu+2}, \alpha_{\rho}=\alpha_{\mu}$ and $\alpha_{i}=\alpha_{\mu-i}, i=1,2,3$ and we have that $\alpha_{\mu-1} \geq \alpha_{\mu-2}>$ $\alpha_{\mu-3}$. By applying the transformation

$$
r^{\prime}=\frac{\omega z \alpha_{\mu+2}-\alpha_{\mu+1}}{\omega z-1}
$$

where a dimensionless variable $r^{\prime}$ through $r=r^{\prime} M_{B H}$ has been introduced. The radii of the (dimensionless) event $\left(r_{+}^{\prime}\right)$ and Cauchy $\left(r_{-}^{\prime}\right)$ horizons are given (In the usual units: $r_{ \pm}=\frac{G M_{\mathrm{BH}}}{c^{2}} \pm$ $\sqrt{\left(\frac{G M_{B H}}{c^{2}}\right)^{2}-\left(\frac{G e^{2}}{c^{4}}+a^{2}\right)}$.) by:

$$
r_{ \pm}^{\prime}=1 \pm \sqrt{1-a^{2}-e^{2}}
$$


We computed analytically the radial integrals in terms of Appell-Lauricella hypergeometric functions [2] :

Theorem 5. The deflection angle $\delta_{e K N}$, of an equatorial unbound light ray in the gravitational field of a Kerr-Newman black hole is computed in closed analytic form in terms of Appell-Lauricella hypergeometric functions [2]:

$$
\begin{aligned}
\delta_{e K N} & :=-\pi+2\left[\frac{-2 A_{+}^{e q K N} \sqrt{\omega}(\alpha-\beta)}{H^{+}} F_{D}\left(\frac{1}{2}, \beta_{\mathbf{3}}^{\mathbf{9}}, \frac{3}{2}, \mathbf{z}_{+}^{\mathbf{r}}\right)\right. \\
& +\frac{2 A_{+}^{e q K N} \sqrt{\omega}(\alpha-\beta)}{H^{+} \kappa_{+}^{\prime 2}}\left(-F_{1}\left(\frac{1}{2}, \beta_{A}^{\mathbf{r a}}, \frac{3}{2}, \mathbf{z}_{\mathbf{A}}^{\mathbf{r}}\right)+F_{D}\left(\frac{1}{2}, \beta_{\mathbf{3}}^{\mathbf{9}}, \frac{3}{2}, \mathbf{z}_{+}^{\mathbf{r}}\right)\right) \\
& +\frac{-2 A_{-}^{e q K N} \sqrt{\omega}(\alpha-\beta)}{H^{-}} F_{D}\left(\frac{1}{2}, \beta_{\mathbf{3}}^{\mathbf{9}}, \frac{3}{2}, \mathbf{z}_{-}^{\mathbf{r}}\right) \\
& \left.+\frac{2 A_{-}^{e q K N} \sqrt{\omega}(\alpha-\beta)}{H^{-} \kappa_{-}^{\prime 2}}\left(-F_{1}\left(\frac{1}{2}, \beta_{A}^{\mathbf{r a}}, \frac{3}{2}, \mathbf{z}_{\mathbf{A}}^{\mathbf{r}}\right)+F_{D}\left(\frac{1}{2}, \beta_{\mathbf{3}}^{\mathbf{9}}, \frac{3}{2}, \mathbf{z}_{-}^{\mathbf{r}}\right)\right)\right] \\
& +2 \Phi \frac{\Gamma(1 / 2) \Gamma(1)}{\Gamma(3 / 2)}\left(\frac{1}{\sqrt{(\gamma-\alpha)(\boldsymbol{\delta}-\alpha)}}\right) F_{1}\left(\frac{1}{2}, \beta_{A}^{\mathbf{r a}}, \frac{3}{2}, \mathbf{z}_{\mathbf{A}}^{\mathbf{r}}\right),
\end{aligned}
$$

The roots of the quartic radial polynomial (3.2), that appear in the closed form solution of Theorem 5, have been computed in a very elegant closed analytic compact form in terms of the elliptic functions $\wp, \wp^{\prime}$ as follows [2]:

$$
\begin{aligned}
\alpha & =\frac{1}{2} \frac{\wp^{\prime}\left(-x_{1} / 2+\omega\right)-\wp^{\prime}\left(x_{1}\right)}{\wp\left(-x_{1} / 2+\omega\right)-\wp\left(x_{1}\right)}, \\
\beta & =\frac{1}{2} \frac{\wp^{\prime}\left(-x_{1} / 2+\omega+\omega^{\prime}\right)-\wp^{\prime}\left(x_{1}\right)}{\wp\left(-x_{1} / 2+\omega+\omega^{\prime}\right)-\wp\left(x_{1}\right)}, \\
\gamma & =\frac{1}{2} \frac{\wp^{\prime}\left(-x_{1} / 2+\omega^{\prime}\right)-\wp^{\prime}\left(x_{1}\right)}{\wp\left(-x_{1} / 2+\omega^{\prime}\right)-\wp\left(x_{1}\right)}, \\
\delta & =\frac{1}{2} \frac{\wp^{\prime}\left(-x_{1} / 2\right)-\wp^{\prime}\left(x_{1}\right)}{\wp\left(-x_{1} / 2\right)-\wp\left(x_{1}\right)}
\end{aligned}
$$

where the point $x_{1}$ is determined from the equation

$$
a^{2}-\Phi^{2}=-6 \wp\left(x_{1}\right)
$$

while the equations

$$
2(a-\Phi)^{2}=4 \wp^{\prime}\left(x_{1}\right),-3 \wp^{2}\left(x_{1}\right)+g_{2}=-e^{2}(\Phi-a)^{2}
$$

determine the Weierstraß invariants $\left(g_{2}, g_{3}\right)$ with the result:

$$
\begin{aligned}
& g_{2}=\frac{1}{12}\left(a^{2}-\Phi^{2}\right)^{2}-e^{2}(\Phi-a)^{2}, \\
& g_{3}=-\frac{1}{216}\left(a^{2}-\Phi^{2}\right)^{3}-\frac{1}{4}(a-\Phi)^{4}-e^{2}(\Phi-a)^{2}\left(\frac{a^{2}-\Phi^{2}}{6}\right) .
\end{aligned}
$$

The rest of the quantities in $\delta_{e K N}$ are defined in [2]. 
Corollary 6. The deflection angle for the non-spinning $(a=\Lambda=0)$ Reisser-Nordström black hole is given by:

$$
\begin{aligned}
\delta_{e R N} & =2 \Phi \frac{\Gamma(1 / 2) \Gamma(1)}{\Gamma(3 / 2)}\left(\frac{1}{\sqrt{(\gamma-\alpha)(\delta-\alpha)}}\right) F_{1}\left(\frac{1}{2}, \beta_{A}^{\mathbf{r a}}, \frac{3}{2}, \mathbf{z}_{\mathbf{A}}^{\mathbf{r}}\right)-\pi \\
& =\frac{4 \Phi}{\sqrt{(\beta-\delta)(\alpha-\gamma)}} \operatorname{sn}^{-1}\left(\sqrt{\frac{\beta-\delta}{\alpha-\delta}}, k^{2}\right)-\pi=\frac{4 \Phi}{\sqrt{(\beta-\delta)(\alpha-\gamma)}} \mathrm{cn}^{-1}\left(\sqrt{\frac{\alpha-\beta}{\alpha-\delta}}, k^{2}\right)-\pi \\
& =\frac{4 \Phi}{\sqrt{(\beta-\delta)(\alpha-\gamma)}} \mathrm{dn}^{-1}\left(\sqrt{\frac{\alpha-\beta}{\alpha-\gamma}}, k^{2}\right)-\pi
\end{aligned}
$$

with $k^{2}:=\frac{\alpha-\delta}{\beta-\delta} \frac{\gamma-\beta}{\gamma-\alpha}$.

Remark 7. The deflection angle for the uncharged $(e=0)$ rotating black hole (Kerr and Kerr(anti) de Sitter) has been investigated in [3].

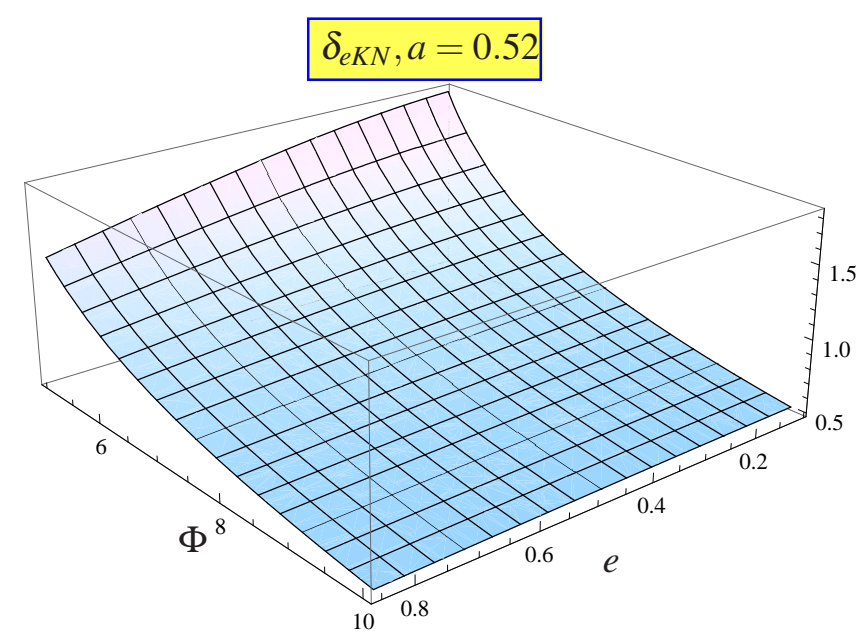

Figure 1: Plot of the deflection angle $\delta_{e K N}$ as a function of the parameters $\Phi, e$ for fixed value for the Kerr parameter (spin) $a=0.52$.

Remark 8. From the 3-d plots of the deflection angle in Theorem 5, Figs.1-2, we observe that the smaller the Kerr parameter the larger the deflection, for fixed values of the parameters $\Phi, e$. We also observe, the strong dependence of the deflection angle on the electric charge, for smaller values of the spin of the black hole, particularly for small values of the impact factor parameter $\Phi$-see Fig.1. For a fixed small distance $\alpha$ there is a strong dependence of $\delta_{e K N}$ on the black hole's electric charge: the larger the electric charge e, the smaller $\delta_{e K N}$. For fixed values for a,e, the deflection angle $\delta_{e K N}$ decreases with increasing values for the parameter $\Phi$. Concerning tentative values for the electric charge of the $\operatorname{SgrA} *$ :

$$
\begin{aligned}
& e=0.11 \sqrt{6.6743 \times 10^{-8}} 4.06 \times 10^{6} \times 1.9884 \times 10^{33} \mathrm{esu}=2.29 \times 10^{35} \mathrm{esu} \Leftrightarrow 7.65 \times 10^{25} \mathrm{C}, \\
& e=0.85 \sqrt{6.6743 \times 10^{-8}} 4.06 \times 10^{6} \times 1.9884 \times 10^{33} \mathrm{esu}=1.77 \times 10^{36} \mathrm{esu} \Leftrightarrow 5.94 \times 10^{26} \mathrm{C}
\end{aligned}
$$




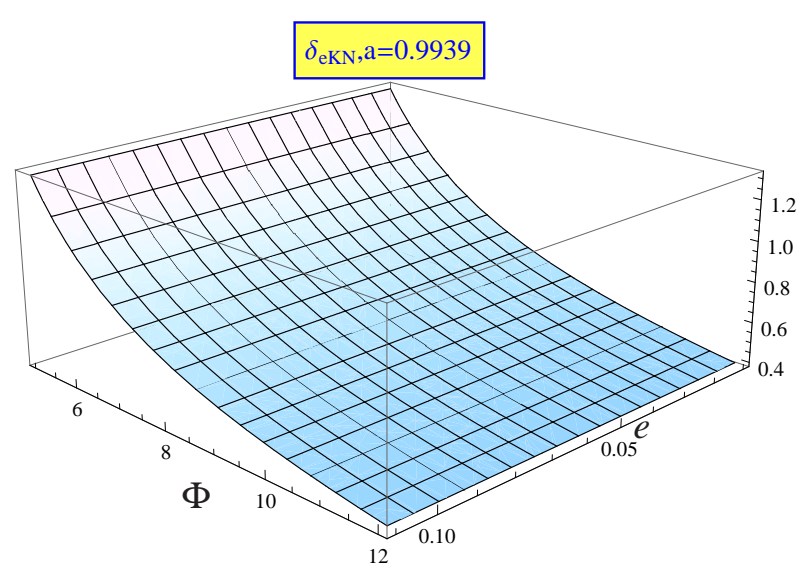

Figure 2: Plot of the deflection angle $\delta_{e K N}$ as a function of the parameters $\Phi, e$ for fixed Kerr parameter $a=0.9939$.

we note that their likelihood is debatable: There is an expectation that the electric charge e trapped in the galactic nucleous will not likely reach so high values as the ones close to the extremals predicted in (1.6) that allow the avoidance of a naked singularity.

\section{Exact analytic solution of the lens equations in the Kerr-Newman spacetime}

The lens equations in this case are Eq. $(1.7)_{1}$ and:

$$
\begin{gathered}
\frac{\mathrm{d} \phi}{\mathrm{d} r}=\frac{a\left(-e^{2}+2 M r\right)}{ \pm \Delta^{K N} \sqrt{R}}+\frac{-a^{2} \Phi}{ \pm \Delta^{K N} \sqrt{R}}+\frac{\Phi}{ \pm \sin ^{2} \theta \sqrt{\Theta}} \frac{\mathrm{d} \theta}{\mathrm{d} r} \\
R:=\left[\left(r^{2}+a^{2}\right)-a \Phi\right]^{2}-\Delta^{K N}\left(\mathscr{Q}+(\Phi-a)^{2}\right), \Theta=\left[\mathscr{Q}+(\Phi-a)^{2}\right]-\frac{\left(a \sin ^{2} \theta-\Phi\right)^{2}}{\sin ^{2} \theta} .
\end{gathered}
$$

We have written the lens Eqs. (1.7) $)_{1}$ and (4.1) in the form [2]:

$$
\begin{aligned}
R_{1}^{K N}\left(x_{i}, y_{i}\right)-A_{1}\left(x_{i}, y_{i}, x_{S}, y_{S}, m\right) & =0, \\
\Delta \phi\left(x_{S}, y_{S}, n\right)-R_{2}^{K N}\left(x_{i}, y_{i}\right)-A_{2}\left(x_{i}, y_{i}, x_{S}, y_{S}, m\right) & =0 .
\end{aligned}
$$

In these equations $n$ denotes the number of windings around the $z$-axis and $m$ the number of turning points in the polar motion.

Theorem 9. The analytic solution of the lens equations for the KN spacetime is given in terms of 
Weierstraßelliptic function and multivariable hypergeometric functions of Appell-Lauricella [2]

$$
\begin{aligned}
R_{1}^{K N}\left(x_{i}, y_{i}\right) & =A_{1}\left(x_{i}, y_{i}, x_{S}, y_{S}, m\right) \Leftrightarrow \frac{2}{\sqrt{(\alpha-\gamma)(\alpha-\delta)}} \frac{\Gamma(1 / 2)}{\Gamma(3 / 2)} F_{1}\left(\frac{1}{2}, \beta_{A}^{r a}, \frac{3}{2}, \mathbf{z}_{\mathbf{A}}^{\mathbf{r}}\right) \\
& =2(m-1) \frac{1}{2|a|} \sqrt{\frac{z_{m}}{z_{m}\left(z_{m}-z_{3}\right)}} \pi F\left(\frac{1}{2}, \frac{1}{2}, 1, \frac{z_{m}}{z_{m}-z_{3}}\right) \\
& +\frac{1}{2|a|} \frac{\sqrt{\left(z_{m}-z_{S}\right)}}{\sqrt{z_{m}\left(z_{m}-z_{3}\right)}} F_{1}\left(\frac{1}{2}, \beta_{A}^{r a}, \frac{3}{2}, \mathbf{z}_{\mathbf{A}}^{\mathbf{1 S}}\right) \frac{\Gamma\left(\frac{1}{2}\right) \Gamma(1)}{\Gamma(3 / 2)} \\
& +\left[1-\operatorname{sgn}\left(\theta_{S} \circ \theta_{m S}\right)\right] \frac{1}{|a|} \frac{\sqrt{\frac{z_{S}\left(z_{m}-z_{3}\right)}{z_{m}\left(z_{S}-z_{3}\right)}}}{\sqrt{z_{m}-z_{3}}} F_{1}\left(\frac{1}{2}, \beta_{A}^{r a}, \frac{3}{2}, \mathbf{z}_{\mathbf{A}}^{\mathbf{2 S}}\right) \\
& +\frac{1}{2|a|} \frac{\sqrt{\left(z_{m}-z_{O}\right)}}{\sqrt{z_{m}\left(z_{m}-z_{3}\right)}} F_{1}\left(\frac{1}{2}, \beta_{A}^{r a}, \frac{3}{2}, \mathbf{z}_{\mathbf{A}}^{\mathbf{1 O}}\right) \frac{\Gamma\left(\frac{1}{2}\right) \Gamma(1)}{\Gamma(3 / 2)} \\
& +\left[1-\operatorname{sgn}\left(\theta_{O} \circ \theta_{m O}\right)\right] \frac{1}{|a|} \frac{\sqrt{\frac{z_{0}\left(z_{m}-z_{3}\right)}{z_{m}\left(z_{0}-z_{3}\right)}}}{\sqrt{z_{m}-z_{3}}} F_{1}\left(\frac{1}{2}, \beta_{A}^{r a}, \frac{3}{2}, \mathbf{z}_{\mathbf{A}}^{\mathbf{2 O}}\right), \\
& -\phi_{S}=R_{2}^{K N}\left(x_{i}, y_{i}\right)+A_{2}\left(x_{i}, y_{i}, x_{S}, y_{S}, m\right), \\
\xi_{S}= & \wp\left(2 \sigma _ { S } \left[R_{1}^{K N}\left(x_{i}, y_{i}\right)\right.\right. \\
& \left.\left.-2(m-1) \frac{1}{2|a|} \sqrt{\frac{z_{m}}{z_{m}\left(z_{m}-z_{3}\right)}} \pi F\left(\frac{1}{2}, \frac{1}{2}, 1, \frac{z_{m}}{z_{m}-z_{3}}\right)+\cdots\right]+\varepsilon\right)
\end{aligned}
$$

where

$$
\begin{aligned}
A_{2}\left(x_{i}, y_{i}, x_{S}, y_{S}, m\right) & =\frac{\Phi}{|a|} \frac{(m-1)}{\sqrt{\left(z_{m}-z_{3}\right)}} \frac{\pi}{\left(1-z_{m}\right)} F_{1}\left(\frac{1}{2}, 1, \frac{1}{2}, 1, \frac{-z_{m}}{1-z_{m}}, \frac{z_{m}}{z_{m}-z_{3}}\right) \\
& +\frac{\Phi}{2|a|} \sqrt{\frac{\left(z_{m}-z_{S}\right)}{z_{m}}} \frac{1}{\sqrt{z_{m}-z_{3}}} \frac{2}{\left(1-z_{m}\right)} F_{D}\left(\frac{1}{2}, \beta_{3}^{4}, \frac{3}{2}, \mathbf{z}_{\mathbf{S}}^{\mathbf{1}}\right) \\
& +\left[1-\sigma_{S}\right] \frac{\Phi}{|a|} \frac{z_{S}}{z_{m}} \frac{z_{S}-z_{m}}{1-z_{S}} \frac{1}{\sqrt{z_{S}\left(z_{S}-z_{m}\right)\left(z_{3}-z_{S}\right)}} \times F_{D}\left(1, \beta_{3}^{7}, \frac{3}{2}, \mathbf{z}_{\mathbf{S}}^{\mathbf{S}}\right) \\
& +\frac{\Phi}{2|a|} \sqrt{\frac{\left(z_{m}-z_{O}\right)}{z_{m}}} \frac{1}{\sqrt{z_{m}-z_{3}}} \frac{2}{\left(1-z_{m}\right)} F_{D}\left(\frac{1}{2}, \beta_{3}^{4}, \frac{3}{2}, \mathbf{z}_{\mathbf{O}}^{\mathbf{1}}\right) \\
& +\left[1-\sigma_{O}\right] \frac{\Phi}{|a|} \frac{z_{O}}{z_{m}} \frac{z_{O}-z_{m}}{1-z_{O}} \frac{1}{\sqrt{z_{O}\left(z_{O}-z_{m}\right)\left(z_{3}-z_{O}\right)}} \times F_{D}\left(1, \beta_{3}^{7}, \frac{3}{2}, \mathbf{z}_{\mathbf{O}}^{\mathbf{2}}\right) \\
\theta_{m O} & :=\operatorname{Arccos}\left(\operatorname{sign}\left(y_{i}\right) \sqrt{z_{m}}\right)=\operatorname{Arccos}\left(\operatorname{sign}\left(\beta_{i}\right) \sqrt{z_{m}}\right)
\end{aligned}
$$

$y_{i}$ is the possible position of the image and:

$$
\theta_{m S}:= \begin{cases}\theta_{m O}, & m \text { odd } \\ \pi-\theta_{m O}, & m \text { even }\end{cases}
$$


Also $\theta_{1} \circ \theta_{2}:=\cos \theta_{1} \cos \theta_{2}, \sigma_{S}:=\operatorname{sign} \theta_{S} \circ \theta_{m S}$ and $\varepsilon$ denotes a constant of integration. The Weierstraß invariants in Eq. (4.7) are defined in (2.8) and (2.4). In establishing (4.7) we used the fact that the sum of the second and third term on the right hand side of Eq.(4.5) can be written as: $\int_{\xi_{S}}^{\xi_{m}}+\left(1-\sigma_{S}\right) \int_{\xi_{0}}^{\xi_{S}}$, where $\left(\xi_{m}, \xi_{0}\right)$ are extremal values of $\xi$; thus, one can separate from it the expression $\sigma_{S} \int_{\xi_{S}}^{\infty} \propto \sigma_{S} \wp^{-1}(\xi)$.

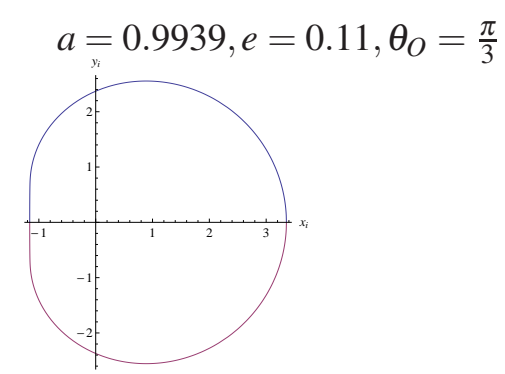

Figure 3: The boundary of the shadow of the KN black hole for $a=0.9939, e=0.11$ for an observer at polar position $\theta_{O}=\pi / 3$.

Conclusion 10. The cosmological constant $\Lambda$ does contribute to the gravitational bending and frame dragging of light by a $K N(A) d S B H$. There is a significant dependance of relativistic observables such as the deflection angle, frame dragging and the periastron precession on the electric charge of the black hole [2]. The shadow of the black hole (see Fig.3 and [2])depends significantly on the electric charge-for fixed values for a, M the larger values for e the smaller the boundary of the shadow of the black hole. Future measurements of the galactic centre black hole and its relativistic observables may constrain significantly or detect the electric charge of the galactic centre rotating black hole as well as the rest of the black hole parameters $M, a, \Lambda$ and will therefore determine the type of the galactic centre black hole.

\section{References}

[1] E. T. Newman et al, Metric of a Rotating, Charged Mass, Jour. of Math. Physics,6,(1965)918; R P Kerr, Gravitational field of a spinning mass as an example of algebraically special metrics, Phys. Rev. Lett. 11(1963) 237; Z. Stuchlík, The motion of test particles in black-hole backgrounds with non-zero cosmological constant, Bull. of the Astronomical Inst. of Chech 34 (1983) 129

[2] G.V. Kraniotis, Gravitational lensing and frame-dragging of light in the Kerr-Newman and the Kerr-Newman-(anti) de Sitter black hole spacetimes, Gen.Rel.Grav.46 (2014) 11, 1818

[3] G.V. Kraniotis, Frame dragging and bending of light in Kerr and Kerr-(anti) de Sitter spacetimes, Clas.Quantum Grav.22 (2005),4391-4424; G. V. Kraniotis, Precise analytic treatment of Kerr and Kerr-(anti) de Sitter black holes as gravitational lenses,Clas. Quantum Grav.28 (2011), 085021

[4] G. Lauricella Sulle funzioni ipergeometriche a più variabili, Rend.Circ.Mat. Palermo 7 (1893) 111-158; P. Appell Sur les fonctions hypergéometriques de deux variables, J. Math.Pure Appl.8 (1882) 173-216 